\title{
A novel strain sensor using a microchannel embedded in PDMS
}

\author{
Angelica Campigotto ${ }^{1}$, Stephane Leahy ${ }^{1,2}$, Ayan Choudhury ${ }^{1}$, Guowei Zhao ${ }^{3}$, Yongjun Lai*1 \\ ${ }^{1}$ Department of Mechanical and Materials Engineering, Queen's University, Kingston, Canada \\ ${ }^{2}$ Soundskrit, Montreal, QC, Canada \\ ${ }^{3}$ School of Astronautics, BeiHang University, Beijing, China
}

Received: May 22, 2018

DOI: $10.5430 /$ jbei.v4n2p1
Accepted: June 21, 2018

Online Published: June 26, 2018

\begin{abstract}
A novel, inexpensive, and easy-to-use strain sensor using polydimethylsiloxane (PDMS) was developed. The sensor consists of a microchannel that is partially filled with a coloured liquid and embedded in a piece of PDMS. A finite element model was developed to optimize the geometry of the microchannel to achieve higher sensitivity. The highest gauge factor that was measured experimentally was 41 . The gauge factor was affected by the microchannel's square cross-sectional area, the number of basic units in the microchannel, and the inlet and outlet configuration. As a case study, the developed strain sensors were used to measure the rotation angle of the wrist and finger joints.
\end{abstract}

Key Words: Strain gauge, PDMS, Microchannel, Flexible

\section{INTRODUCTION}

There are several methods of measuring strain. The most common method is using strain gauges, which are typically electrical devices that change resistance in proportion to the amount of strain they are exposed to. ${ }^{[1]}$ Currently, the majority of strain gauges use foil gauges to produce an electrical signal. ${ }^{[2]}$ The bonded metallic stain gauge is the most commonly used, consisting of fine wires arranged in a grid pattern. Usually the metallic strain gauges have a gauge factor around 2. ${ }^{[3]}$

Recently, a single optical sensor, created from unaltered telecom-grade optical fiber, is reported for measuring complex strain responses. ${ }^{[4]}$ Strain gauges require careful selection and use of a bridge circuit, signal conditioning, wiring, and data acquisition unit to function properly. The more com- plex the device the more expensive the system, with most strain gauges ranging between $\$ 20-\$ 400 .{ }^{[5]}$

Digital image processing is a method that utilizes images captured by digital photography devices, ${ }^{[6]}$ such as digital cameras or charge-coupled devices. Digital image processing is an accurate measurement system to examine the characteristics and parameters of an image. Image processing has been used to advance conventional strain measuring techniques since $2001^{[6,7]}$ and recently is being used in a variety of applications. ${ }^{[6,7]}$

In this paper, we present a novel polydimethylsiloxane (PDMS)-based strain sensor assisted by an image processing technique. Such sensor is highly flexible and could conform to multiple, unique surfaces while still having a high gauge factor and large dynamic range.

\footnotetext{
*Correspondence: Yongjun Lai; Email: lai@queensu.ca; Address: Department of Mechanical and Materials Engineering, Queen’s University, Kingston, ON, K7L 3N6, Canada.
} 


\section{DESIGN AND FABRICATION}

\subsection{Working principle}

The strain sensor is composed of a flexible PDMS base with an embedded microchannel at its surface and a PDMS top that covers the microchannel. The microchannel has multiple
L-units that form a zig-zag, an indicator channel, as well as an inlet and an outlet exposed to the atmosphere. The microchannel is filled with a coloured indicator liquid. Figure 1 shows the design of the sensor with a detailed view of a single basic unit: L-unit (see Figure 1 left).

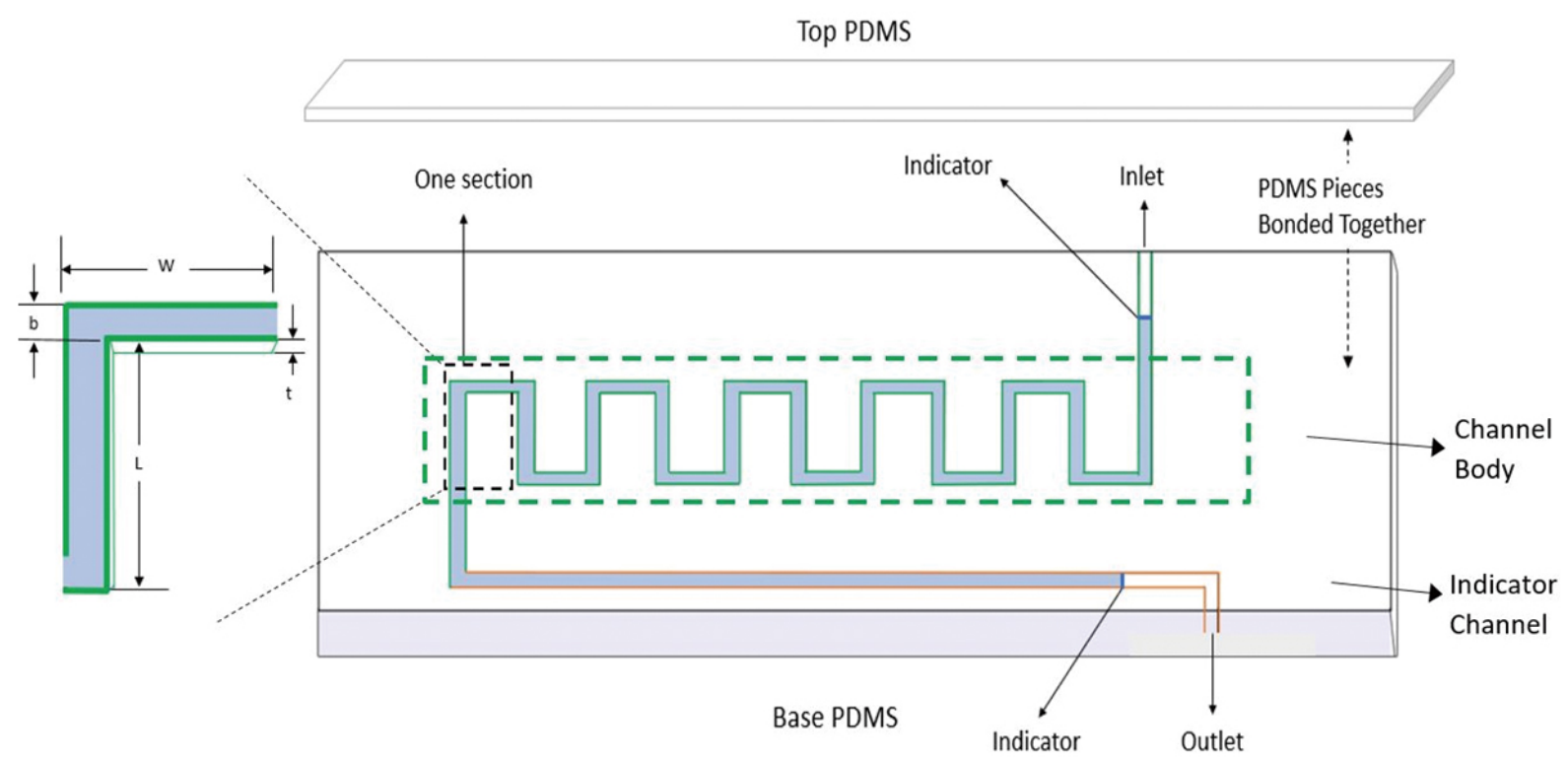

Figure 1. Schematic of the PDMS sensor with the outlines microchannel. The microchannel is filled with indicator liquid shown in blue. The "body" of the microchannel is outlined in green while the "indicator channel" is highlighted in orange. The inlet and outlet of the system is labeled. The indicator is labeled and highlighted with a thick blue line; the indicator is the junction between the end of the liquid and the air within the channel. A square black dashed line illustrates a single L-unit. A larger view of a single L-unit can be shown on the left-hand side with labeled width (w), length (L), microchannel base (b), and thickness (t).

Since PDMS is adhesive, the sensor can be attached to a surface. As the surface deforms, the sensor will deform simultaneously, creating a volume change in the microchannel. Since the colored indicator liquid in the microchannel is incompressible, the volume change of the microchannel will displace the liquid. The movement of the liquid in the microchannel is directly associated with the strain of the system. A cellphone camera and image processing program are used to track the motion of the indicator liquid.

Depending on the composition of the PDMS used to fabricate the sensor, the adhesiveness and mechanical flexibility of the sensor can be tuned for the desired application. For example, this could include measuring the strain of irregular surfaces or measuring very high strain, which would be difficult to do with conventional strain gauges.

\subsection{Optimization}

To optimize the geometry of the microchannel, a finite element analysis was performed with ANSYS. A simple design, representative of the main sensing concept, was considered. A PDMS base of $50 \times 15 \times 1 \mathrm{~mm}^{3}$ and a microchannel with 10 L-units with a default length of $4 \mathrm{~mm}$, width of $1.6 \mathrm{~mm}$, and a microchannel base and thickness of $80 \mu \mathrm{m}$ was used. The indicator channel was kept in line with the body of the microchannel so that symmetric deformation along the $x$ - and $z$-directions could be assumed. A tensile stress of $0.133 \mathrm{MPa}$ was applied to both ends of the system in the $x$-direction (see Figure 2).

As the PDMS base is elongated, the PDMS width decreases. Since the thickness of the PDMS base is much smaller than its width and length, the deformation in the thickness direction is insignificant and can be neglected in the evaluation of volume change. Therefore, the section of the L-unit (with or length) that undergoes the largest area change will dominate the motion of the indicator liquid. 


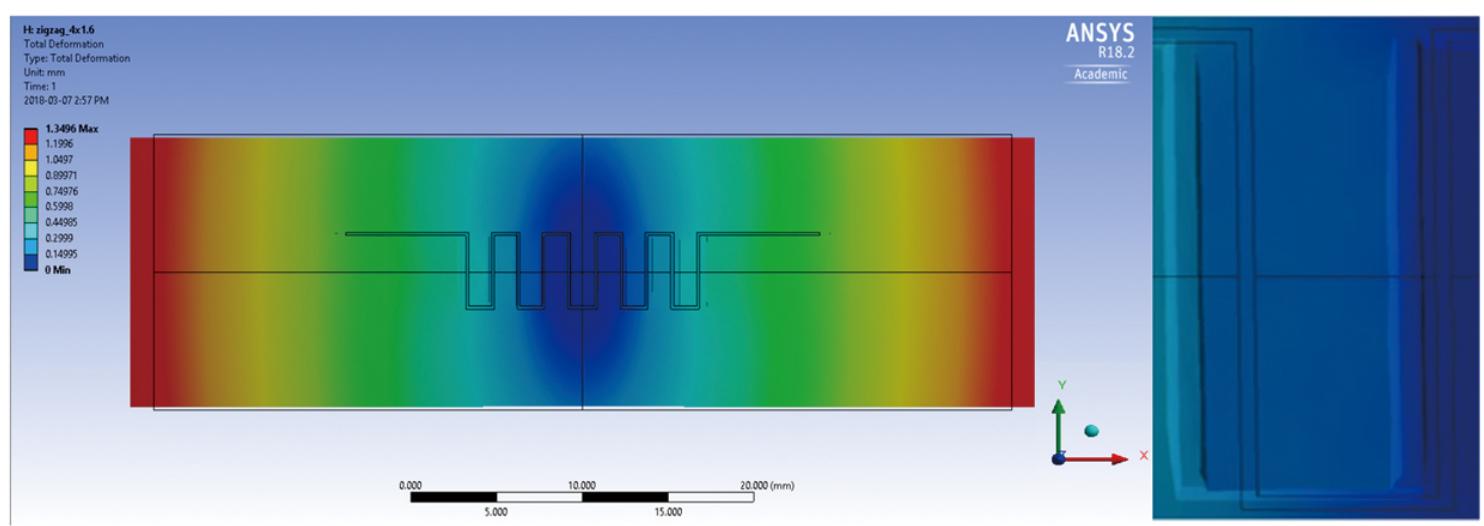

Figure 2. Micro-sensor design modeled in ANSYS with a tensile stress of $0.133 \mathrm{MPa}$ applied on either end of the surface in the $x$-direction. The original shape is outlined in black and the final deformed is shown in contouring bands as a solid shape. On the right, a close-up of the deformation of the micro-channel can be viewed. The sections parallel to the deformation decreased in size while the sections perpendicular to the deformation increase in size.

\subsubsection{Microchannel size}

The square internal microchannel was varied and compared in ANSYS. The microchannel base and thickness were kept equal at sizes of $180,140,100$, and $60 \mu \mathrm{m}$. To simplify the analysis, only the area in the $x-y$-direction was analysed, as the $z$-direction was determined to be negligible to the overall deformation of the system.

As the size of the microchannel base decreased, the initial area before deformation $\left(A_{0}\right)$ and the final area after deformation $\left(A_{1}\right)$ of the microchannel decreased. However, the change in the area of the microchannel $\left(A_{0} / A_{1}\right)$ increased (see Figure 3 ). Therefore, to increase the sensor sensitivity, the indicator channel size should be small.

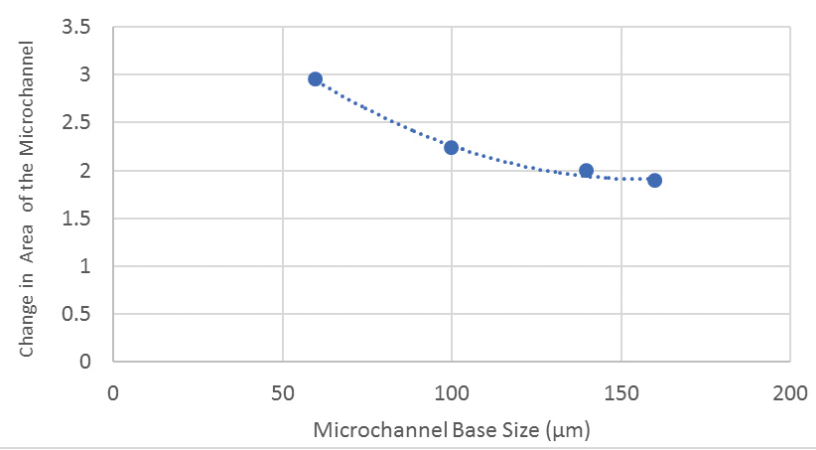

Figure 3. Change in microchannel base $(\mu \mathrm{m})$ plotted against the change in area $\left(A_{0} / A_{1}\right)$ of the microchannel

\subsubsection{Length and width of L-unit}

The ratio between the length and width of a single L-unit was studied in terms change in area of the microchannel. An optimal length-to-width ratio was determined by running different simulations with varying widths to a constant length Published by Sciedu Press and determining the largest change in microchannel area (see Figure 4).

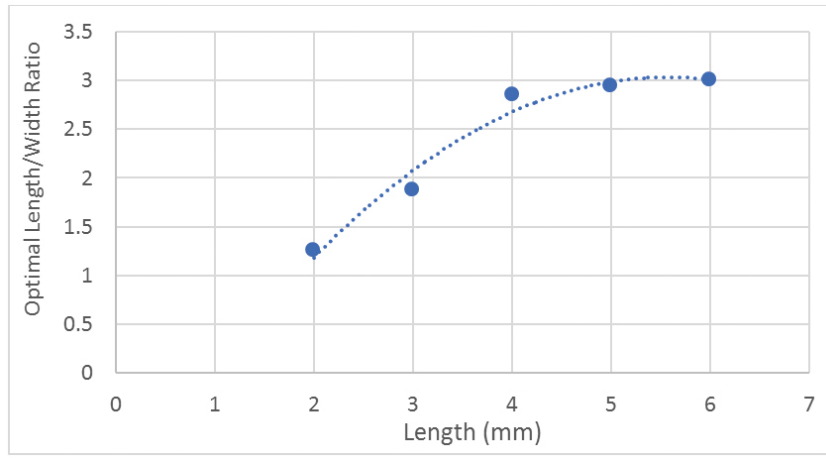

Figure 4. Optimization of the length and width ratio resulting in the largest change in area of the microchannel

Figure 4 shows the optimal length-to-width ratio at given lengths. For example, when the length is $6 \mathrm{~mm}$, the width should be $2 \mathrm{~mm}$ to create a L-unit that gives the largest change in microchannel area after pressure is applied. As the length of the L-unit increases, the optimal length-to-width ratio increases up to a ratio of about 3 .

\subsection{Fabrication}

A high-speed micro CNC milling machine was used to manufacture a mold in which the sensor base was cast with PDMS. The PDMS pre-polymer and curing agent were mixed at a ratio of 10:1 and poured into the mold. ${ }^{[8]}$ The mold was placed in a vacuum chamber for $40 \mathrm{~min}$ for degassing, until all the air bubbles had disappeared. The mold was placed into the oven at $50^{\circ} \mathrm{C}$ for 40 minutes and was cooled to room temperature and then the sensor body was released from the mold. A thin top layer was created by spin coating PDMS on a flat 
surface and then curing the PDMS in the oven at $50^{\circ} \mathrm{C}$ for 30 minutes. ${ }^{[9]}$ The two pieces of PDMS were surface treated using a Corona Treater, from Electro-Technic Products Inc., then bonded together. ${ }^{[10]}$ Dyed water, the indicator fluid, was then injected into the microchannel. Dyed water, the indicator fluid, was then injected into the microchannel. Water was used for its low viscosity and ability to be dyed bright colours. However, PDMS is permeable to water so if left for a long period of time or under high temperature the water within the channel would diffuse out of the micro-channel and evaporate into the atmosphere. Under of temperatures of $0^{\circ} \mathrm{C}$ or below the water will freeze and not properly function. Different incompressible fluids with low freezing points and impermeability to PDMS, such as oils, will be investigated in the future.

\section{EXPERIMENTAL TESTING}

\subsection{Linear testing}

Experimental testing was conducted using the TA.XTPlus Texture Analyzer. The main objective of the experimental testing was to determine the relationship between the deformation of the PDMS and the movement of indicator fluid. The experimental setup is shown in Figure 5.

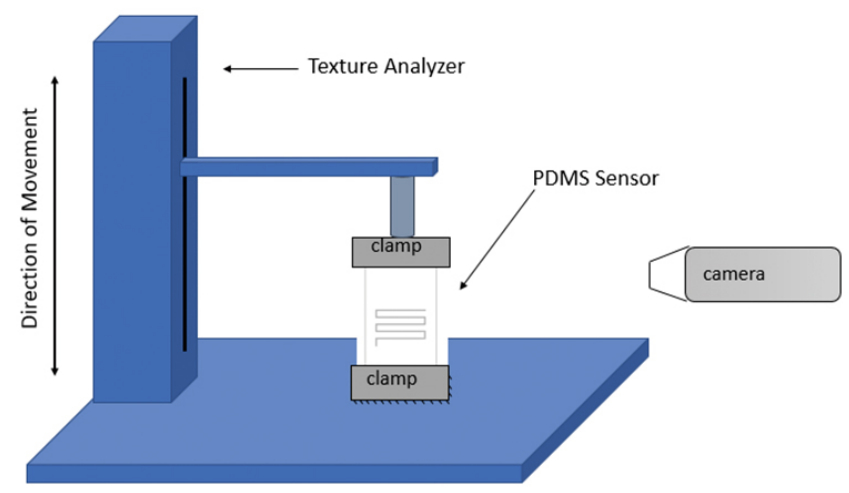

Figure 5. Experimental setup, using a Micro Texture Analyzer. The PDMS was placed in between two clamps with the one clamp fixed to the ground and another able to move freely in the vertical direction. A microscope connected to a laptop was used to record the movement of the indicator fluid as the PDMS was deformed at a uniform rate.

The sensor was stretched at a constant rate of 10 seconds $/ \mathrm{mm}$ while the movement of the indicator liquid was recorded by a camera through a microscope. An image processing program was written to determine the displacement of the indicator liquid.

\subsection{Human joint monitoring}

The need for a continuous human joint monitoring system is increasing in the market due to the benefits of tracking the angle of the joint throughout the day. Human joint motion is often monitored in the medical and biomechanical fields to study the functionality and mechanical behaviour of the structure. Physicians will study a patients' joint motion in cases of rehabilitation of disabilities such as osteoarthritis, joint implants, ligament injury and more. Generally, physicians are only able to track the motion of the joint during in office examinations. These environmentally controlled situations do not realistically portray the way the joint behaves in the patients' daily life. Therefore, a continuous monitoring system for tracking the motion of a targeted joint is essential for a reliable assessment of the patients' disability. Knowledge can be obtained on the behaviour of the joint and the amount of use allowing for an improved, tailored rehabilitation program to be created for each patient, as well a constant monitoring system during the at home rehabilitation process. ${ }^{[10,11]}$

A novel idea was created in order to use PDMS with an internal micro-sensor that will be flexible enough to monitor the deformation of an irregularly shaped system when deformed multiple directions. The PDMS is optically transparent and can be adhered directly onto the skin. Since the device is small and able to adhere directly onto the skin, the user can comfortably wear the device all day without interfering with the joint's movement.

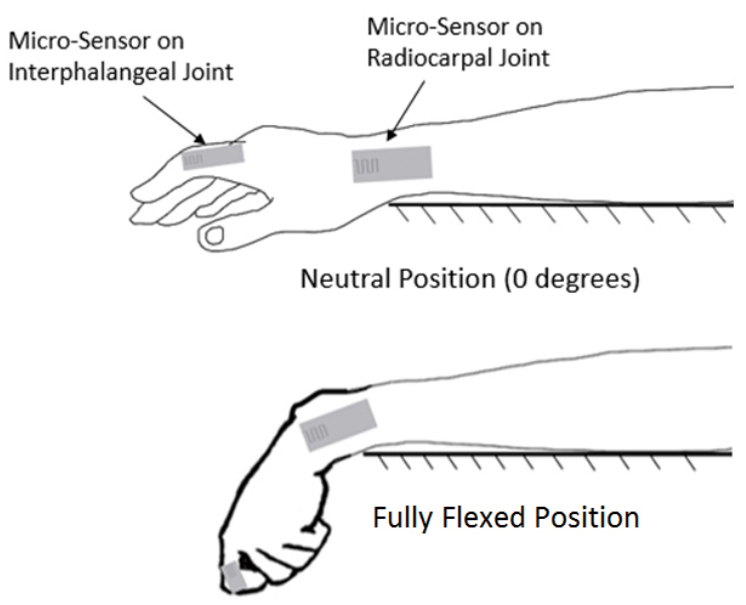

Figure 6. The Micro-sensor was placed on the interphalangeal and radiocarpal joint and tested at various angles between the neutral position $\left(0^{\circ}\right)$ to the fully flexed position $\left(100^{\circ}\right.$ for interphalangeal joint and $85^{\circ}$ for radiocarpal joint). The angle of the joint was set at specific points and the indicator fluid movement was then captured using a camera and post processed with visual software. 
The device can give an instantaneous visual feedback of the motion of the joint. ${ }^{[12]}$ Having a visual feedback system can allow the patients and physicians to track the progression of the disability easily and quickly over time.

The interphalangeal joint and radiocarpal joint were tested using the PDMS micro-sensor. The PDMS was adhered onto the skin and as the joint flexed to various angles; an image of the indicator fluid moving in the microchannel was obtained at each joint position. Figure 6 illustrates the joint movement at the neutral position and the fully flex position.

\section{RESULTS AND DISCUSSION}

\subsection{Linearity testing}

\subsubsection{Microchannel size}

An experimental test similar to the ANSYS simulation was conducted. The base of the sensor was $30 \mathrm{~mm} \times 15 \mathrm{~mm} \times$ $1 \mathrm{~mm}$ the microchannel had $6 \mathrm{~L}$-units with a length of $4 \mathrm{~mm}$ and a width of $1.6 \mathrm{~mm}$ and varying the microchannel's square cross-section area. As predicted in the simulation, as the microchannel base and thickness were decreased from $180 \mu \mathrm{m}$ to $60 \mu \mathrm{m}$, the movement of the indicator fluid increased (see Figure 7). In the Figures 7, 8 and 9, the $x$-axis has an error of $0.001 \mathrm{~mm}$ for the TA.XTPlus Texture Analyzer. The error bars are plus and minus of one pixel for the $y$-axis, which results in $\mathrm{a} \pm 0.059 \mathrm{~mm}$ error.

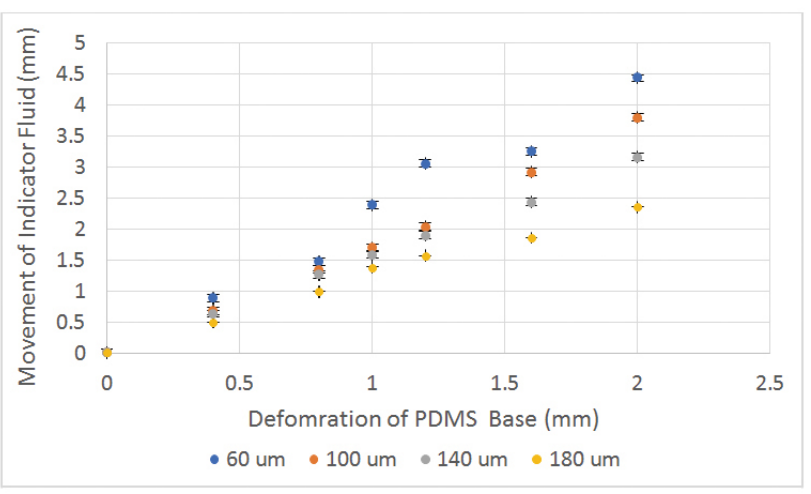

Figure 7. Deformation of PDMS sensor base versus movement of the indicator fluid for a microchannel base and thickness of $60 \mu \mathrm{m}, 100 \mu \mathrm{m}, 140 \mu \mathrm{m}$ and $180 \mu \mathrm{m}$

The images of the indicator with a microchannel base and thickness of $60 \mu \mathrm{m}$ had significant pixilation. This increased the measurement error of the indicator liquid when using the image processing program with a cellphone camera. That is why the slope of the $60 \mu \mathrm{m}$ in Figure 7 is not linear. Using a better camera, a better image processing program, or another liquid measurement system could overcome this problem.

\subsubsection{Number of L-units}

When more L-units are included in the microchannel, the total volume change is expected to increase, forcing the indicator to move more. A PDMS base of $50 \mathrm{~mm} \times 20 \mathrm{~mm}$ $\times 1.5 \mathrm{~mm}$ with an internal microchannel having a constant body of $15 \mathrm{~mm} \times 3 \mathrm{~mm} \times 0.16 \mathrm{~mm}$ and varying number of L-units and indicator microchannel cross-sectional areas were tested. Results were illustrated in Figure 8.

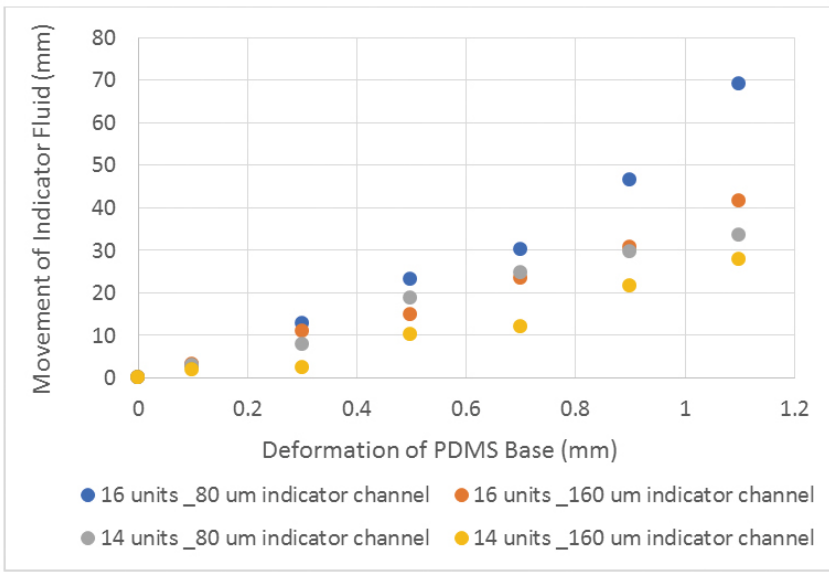

Figure 8. Deformation of the PDMS (mm) plotted against the movement of the indicator fluid $(\mathrm{mm})$ for varying number of sections. Increasing the number of sections increases the initial volume of the channel and thus increases the sensitivity. However, a smaller microchannel base and thickness also increases the indicator fluid sensitivity; so, an optimal design is to increase the number of sections in the body with decreasing the size of the indicator channel.

As can be seen above, adding more L-units resulted in increased movement of the indicator liquid. As well, the 16 and 14 L-units with an indicator channel of $80 \mu \mathrm{m}$ have a better gauge factor than the 14 and $16 \mathrm{~L}$-units with an indicator channel of $160 \mu \mathrm{m}$. It can also be noticed that having 16 L-units with a body and indicator channel size of $160 \mu \mathrm{m}$ has very similar results to the $14 \mathrm{~L}$-units sensor with a body size of $160 \mu \mathrm{m}$ and an indicator channel size of $80 \mu \mathrm{m}$. Overall, increasing the initial volume of the system by adding more L-units improves the sensitivity of the indicator.

\subsubsection{Inlet and outlet configuration}

The sensor relies on a change in the microchannel volume to create a displacement of the indicator liquid. If either or both the inlet and outlet of the channel are sealed then the resulting air pressure within the channel will increase or decrease with the changing channel volume, thus affecting the movement of the indicator fluid. Testing of the inlet and outlet configuration was performed using a sensor with 6 
L-units with a length of $4 \mathrm{~mm}$ and a width of $1.67 \mathrm{~mm}$, and a microchannel base and thickness of $80 \mu \mathrm{m}$.

When both the inlet and outlet are sealed, the movement of the indicator fluid is noticeably lessened. The indicator fluid still moves likely because air is compressed within the microchannel or air leaks through the micropores in the PDMS

\subsection{Human joint monitoring}

Testing was completed on the interphalangeal and radiocarpal joints with the strain sensor. The arm of the test subject was kept flat on a table, in a neutral position (see Figure 9), with the specific joint free to flex and extend. The interphalangeal and radiocarpal joints were measured from a netrual position to their respective fully flexed positions of 0 to $100^{\circ}$ and 0 to $85^{\circ}$, respectively. The joint range was detemined for the test subject piror to the experimentation and will vary based on the subject's joint flexibility. As the angle of the interphalageal and radiocarpal joints began to flex, the indicator fluid began to travel along the microchannel, displaying a linear relationship between the angle of the joint and the position of the indicator liquid, as shown in Figure 10. Testing was completed with a $30 \mathrm{~mm} \times 10 \mathrm{~mm} \times 1.5 \mathrm{~mm}$ sensor base and a microchannel with $10 \mathrm{~L}$-units with a length of $4 \mathrm{~mm}$ and a width of $2 \mathrm{~mm}$, and a microchannel base and thickness of $80 \mu \mathrm{mm}$. This configuration was choosen because its smaller size allowed for an easier fit on the test subject.

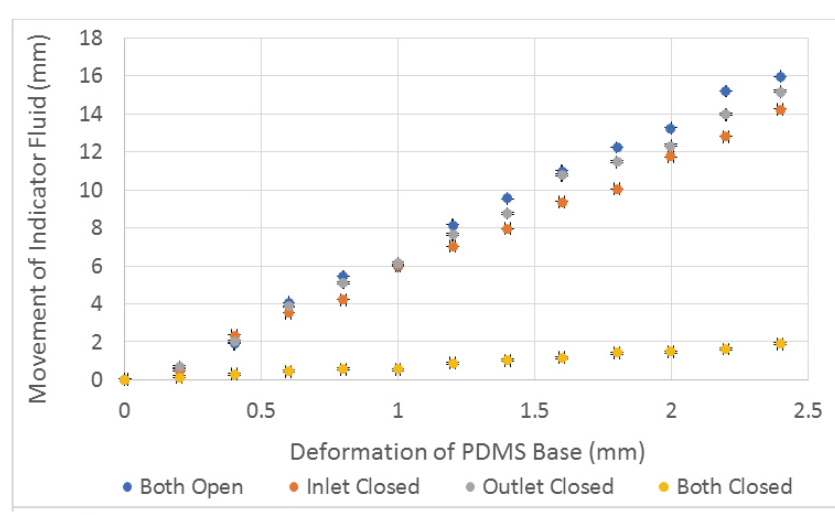

Figure 9. Total indicator fluid movement when the inlet is closed, outlet is closed, both are closed and both are open.

These results demonstrate that the flexion and extension of the interphalangeal and radiocarpal joint can be monitored using the strain sensor. The interphalangeal joint has a stronger linear trend when compared to the radiocarpal joint. This is because of the increased error during image processing when determining the angle of the radiocarpal joint. The interphalangeal joint is comprised of just 2 bones that form a hinge joint, whereas the radiocarpal joint is comprised of up to 15 bones that form a condyloid type joint. This additional complexity of the radiocarpal join leads to higher angular error.

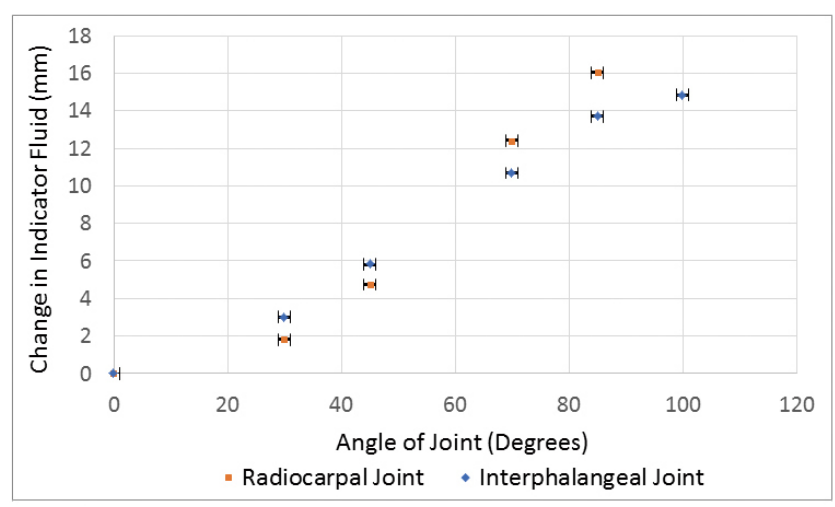

Figure 10. Joint angle plotted against the changing location of the indicator fluid, in $\mathrm{mm}$. The radiocarpal joint is shown in orange while the Interphalangeal joint is shown in blue. The $x$-axis has an error of \pm 1 degree and the $y$-axis has an error of $\pm 0.059 \mathrm{~mm}$ for both the radiocarpal and interphalangeal joint.

\section{Conclusion}

A novel PDMS strain sensor was proposed to measure the deformation of surfaces. It relies on a microchannel enclosed in a PDMS base. The microchannel is filled with an indicator liquid and when the PDMS base deforms, the indicator liquid moves. The motion of the indicator liquid can be captured with a cellphone camera and the image can be processed to determine the deformation of the surface on which the strain sensor is attached.

Experiments showed that the microchannel size, the number of L-units, and the inlet and outlet configuration influenced the motion of the indicator fluid and thus the sensitivity of the sensor. Decreasing the indicator channel's square crosssection area (base and thickness), increasing the initial volume of the microchannel by adding more L-units, and using an open inlet and outlet configuration provided the highest sensitivity.

For a few cents a strain gauge with a gauge factor of 41 can be manufactured. This simple design can be created in a variety of different compositions and forms for an optimal, cost-effective sensor. The flexibility of the PDMS material coupled with the sensors ability to read large dynamic ranges of displacement make it ideal for a wide variety of applications.

A case studied was performed by using the strain sensor to track the angle of the radiocarpal and interphalangeal joints. 
A linear relationship was obtained between the angle of the joints and the position of the indicator fluid. This demonstrates that the PDMS sensor is an effective tool to quickly and accurately track irregularly shapes and deformations, such as joint movement. This could greatly benefit physicians and patients in the rehabilitation process after joint injury.

\section{REFERENCES}

[1] National Instruments. Measuring Strain with Strain Gages. 04 November 2014 [Internet]. Available from: http: //aeweb.tamu.edu/aero214/Measuring\%20Strai n\%20with\%20Strain\%20Gages.pdf [Accessed 2018].

[2] Seika M, Sugiura M, Yamaoka A, et al. An application of computer image-processing and film replica technique to a nickel foil strain gage. Experimental Mechanics. 1997; 37(2): 169-174. https : //doi.org/10.1007/BF02317855

[3] National Instruments Corporation. Measuring Strain with Strain Gages. National Instruments. 2018. [Internet]. Available from: http: //www.ni.com/white-paper/3642/en/ [Accessed 06 2018].

[4] Luna. Distributed Fiber Optic Strain Sensing: Applications in Composites Test and Measurement. Luna, July 2013. [Internet]. Available from: http://lunainc.com/wp-content/uploads /2013/09/LT_TD_EN-FY1317_DFS-Composite-Application s_Rev-3.pdf [Accessed 2018].

[5] omega. Strain Gage. omega; 2018. [Internet]. Available from: https://www .omega.ca/guides/straingages.html?gclid $=$ CjwKCA jws J3ZBRBJEiwAtuvtlMx3iZlnKzJ3ZT0sb5PAhqPZj BM9sbjR00S9QC5k-c5BaSB0JnqfRxoC7h4QAvD_BwE [Accessed 06 2018].

[6] Sutradhar A, Park J, Carrau D, et al. Experimental validation of 3D printed patient-specific implants using digital image correlation and finite element analysis. Computers in Biology and Medicine. 2014;
52: 8-17. PMid:24992729. https://doi.org/10.1016/j.comp biomed.2014.06.002

[7] Vogel JH, Lee D. An Automated Two-View Method for Determining Strain Distributions on Deformed Surfaces. Journal of Materials Shaping Technology. 1989; 6(4): 205-216.

[8] Kawun P, Leahy S, Lai Y. A thin PDMS nozzle/diffuser micropump for biomedical applications. Sensors and Actuators A: Physical. 2016; 249: 149-154. https://doi.org/10.1016/j.sna.2016.08.03 2

[9] Tarigopula V, Hopperstad OS, Langseth M. A study of localisation in dual-phase high-strength steels under dynamic loading using digital image correlation and FE analysis. International Journal of Solids and Structures. 2008; 45(2): 601-619. https ://doi.org/10.101 $6 / j$. ijsolstr.2007.08.021

[10] Electro Technic Products. BD-20AC Laboratory Corona Treater Electro Technic Products. 2018. [Internet]. Available from: http://www.electrotechnicproducts.com/bd-20ac-lab oratory-corona-treater [Accessed 2018].

[11] Gibbs PT, Asada HH. Wearable Conductive Fiber Sensors for MultiAxis Human Joint Angle Measurements. Journal of Neuroengineering. 2005; 2: 2-7. PMid:15733322. https://doi.org/10.1186/ 1743-0003-2-2

[12] Lee C, Take WA, Hoult NA. Optimum Accuracy of Two-Dimensional Strain Measurements Using Digital Image. Journal of Computing in Civil Engineering. 2012; 26(6): 795-803. https ://doi .org/10.1 061/ (ASCE) CP. 1943-5487.0000182 